\title{
L'enseignement - apprentissage du français aux élèves rejoignants
}

\section{Annie Beaucourt}

\section{(2) OpenEdition}

1 Journals

Édition électronique

URL : http://journals.openedition.org/trema/2187

DOI : 10.4000/trema.2187

ISSN : 2107-0997

\section{Éditeur}

Faculté d'Éducation de l'université de Montpellier

\section{Édition imprimée}

Date de publication : 1 juin 1995

Pagination : 57-63

ISSN : 1167-315X

\section{Référence électronique}

Annie Beaucourt, «L'enseignement - apprentissage du français aux élèves rejoignants », Tréma [En ligne], 7 | 1995, mis en ligne le 23 septembre 2013, consulté le 01 mai 2019. URL : http:// journals.openedition.org/trema/2187 ; DOI : 10.4000/trema.2187

Ce document a été généré automatiquement le 1 mai 2019.

Trema 


\title{
L'enseignement - apprentissage du français aux élèves rejoignants
}

\author{
Annie Beaucourt
}

1 Cet article est le fruit d'un travail de terrain de plusieurs années mené auprès d'élèvesrejoignants, dans un collège de Montpellier. Cette expérience nous a permis de repérer un certain nombre de difficultés et de tracer des pistes.

2 L'insuffisance des compétences en français est souvent vécue comme un obstacle majeur à la poursuite d'un cursus scolaire satisfaisant. Chacun sait que les enfants de migrants ne sont pas les seuls à souffrir de déficiences linguistiques, que la non maîtrise de la langue est une raison majeure de l'échec scolaire des enfants dits "en difficulté ». Et les difficultés ne sont pas les seules «difficultés » de l'enfant. Elles sont aussi les difficultés de l'enseignant et celles de l'institution scolaire. ${ }^{1}$

3 Le problème linguistique se pose immédiatement pour les élèves rejoignants. Quand leur présence est prise en compte, les modalités de leur scolarisation diffèrent beaucoup suivant les collèges, sans que ces choix aient toujours un fondement pédagogique :

- soit les élèves sont intégrés dans une classe d'accueil où un enseignant détaché conduit l'enseignement-apprentissage du français, la remise à niveau des élèves et leur intégration progressive dans les classes de niveau où ils sont inscrits. ${ }^{2}$ Dans les établissements les mieux dotés, l'enseignant reçoit le soutien d'un professeur de FLE et d'un animateur. ${ }^{3}$

- soit les élèves intègrent directement leur classe de niveau et bénéficient de quelques heures hebdomadaires de français dispensées par un professeur de FLE. Les deux solutions, structures spécifiques plutôt fermées ou dispositifs plus ouverts, comportent des avantages et des inconvénients, pour l'enseignant et pour l'élève.

4 Un des problèmes tient à la connaissance qu'a le professeur de l'élève lui-même et à celle de son vécu. Un autre a trait à la formation de l'enseignant, à sa connaissance des objectifs scolaires à atteindre et des moyens d'y parvenir. Il tient également aux représentations que se fait l'enseignant de cette population scolaire. 


\section{Langues, représentations et enseignement- apprentissage}

\subsection{L'élève-rejoignant}

Quelques constats s'imposent.

- L'adolescent ${ }^{4}$ qui arrive en France subit une double déchirure. La première est d'ordre affectif. La seconde est d'ordre culturel : l'adolescent passe d'une société à une autre.

- Un autre choc est pour certains non moins capital : c'est celui de la rencontre avec l'école.

6 La langue de communication qu'un enseignement-apprentissage du français pose actuellement comme prépondérante prend déjà, dans le cas des élèves-rejoignants, une dimension informative et structurante autre que pour l'élève étranger du même âge, apprenant le français dans son pays en tant que langue étrangère.

Le français prend nécessairement une dimension cognitive. Pour lui, le français n'est pas une langue étrangère, $c^{\prime}$ est une langue seconde. ${ }^{5}$

8 Le rejoignant va devoir s'adapter et intégrer d'autres formes de langage. En effet, sa vie en France ne s'organise pas uniquement dans le monde de l'école. L'apprentissage du français n'est pas limité au français standard, ni à la langue scolaire. Si, comme le souligne J. GUMPERZ ${ }^{6}$, le travail de repérage des codes n'est pas accompli, la langue que le rejoignant va acquérir se fossilisera en un « interlecte » mêlant les bribes des différentes langues et sociolectes avec lesquels il aura été en contact.

\subsection{Langue, langages et conduites langagières}

\subsubsection{La langue maternelle}

9 Elle continuera à être la langue de communication privilégiée. Elle est donc langue de culture, au sens ethnologique du terme. Comme nous le signalions supra, plus la scolarisation a été poussée et plus cette langue joue en tant qu'élément structurant des connaissances et donc du sujet.

\subsubsection{La langue des pairs}

On appellera ici « langue des pairs » la langue pratiquée par les membres d'un groupe de préférence de même origine ethnique plus encore que nationale, cousin ou fils d'ami.

11 De fait, la langue parlée entre pairs est un syncrétisme, souvent assez hermétique aux non initiés, de français familier à syntaxe simplifiée, de langue maternelle, d'emprunts à d'autres langues - à l'anglais, par exemple, mais pas exclusivement, de verlan, d'argot, de régionalismes et expressions particulières, gestes propres à une période, au groupe luimême. Pour les rejoignants, elle n'est pas facile à vivre car difficile à saisir. C'est la « langue de la rue ». 


\subsubsection{Le français standard}

Il s'agit du français, plus ou moins soutenu, de la communication courante, celui de la télévision mais aussi de la communication officielle avec les administrations, donc langue de communication et langue d'information.

\subsubsection{Le français de l'école}

13 A la fois langue de communication et langue de scolarisation, ce français répond à des codes différents selon les moments, les personnes à qui l'on s'adresse, les thèmes abordés. L'école est sans doute le lieu où la nécessité d'un transcodage rapide s'avère le plus nécessaire, les enjeux communicationnels étant soumis, explicitement ou non, à une évaluation non seulement pédagogique, mais aussi sociale. Ceci dit, il est évident que le français le plus délicat à acquérir - non seulement pour les élèves-rejoignants, d'ailleurs est bien la langue scolaire stricto sensu.

L'enseignement-apprentissage du français aux élèves-rejoignants passe donc par deux phases :

- une première étape au cours de laquelle le français est certainement plus à enseigner en terme de langue étrangère que de langue seconde ;

- une deuxième phase qui sera prise en charge non seulement par l'enseignant responsable de la classe d'accueil ou le professeur de FLE, mais également par les enseignants des différentes matières, quand les élèves rejoignent réellement les cours de leur classe de niveau. Il s'agit alors d'amener l'élève à s'approprier le français en tant que langue de travail, outil de conceptualisation.

Cependant, sur le terrain, des obstacles se dressent empêchant la mise en œuvre correcte et efficace de l'enseignement-apprentissage du français langue seconde, notamment ceux concernant la formation des enseignants d'une part, leurs croyances et représentations d'autre part, croyances attachées au déterminisme économique, à l'impossible adaptation sociale, vu le faible degré de scolarisation antérieure.

16 L'enseignant aura tendance à ne proposer aux rejoignants que des activités impliquant la mise en place d'une langue concrète, qui ne lui donnent pas les moyens d'atteindre le niveau d'abstraction linguistique requis pour conceptualiser les savoirs et les savoir-faire de niveau supérieur nécessaires à la poursuite des études.

17 Or, l'enseignement-apprentissage du français langue seconde se doit de viser à la fois des objectifs communicatifs et linguistiques et des objectifs de structuration des savoirs et des savoir-faire.

\section{Enseignement-apprentissage du français langue 2 : quelques propositions}

L'extrême hétérogénéité des niveaux des élèves interdit un enseignement identique pour tous ; certaines activités concernent cependant l'ensemble de la classe. 


\subsection{Les activités collectives}

19 Un certain nombre d'activités peuvent être menées de façon collective. C'est le cas de la communication orale, dont la démarche sera inspirée dans un premier temps de l'approche communicative, mais devra vite se construire également) autour des acquis langagiers extrascolaires, de façon à les systématiser et à les replacer dans leur contexte situationnel. Cela permet de construire la compétence de communication, mais aussi d'aborder les registres de langue et de mettre en place le nécessaire travail de reformulation.

Il est en effet important de soumettre l'élève à un bain langagier riche, diversifié, qui peut excéder de loin ses capacités de mémorisation et de réinvestissement langagier.

Des exercices systématiques de discrimination auditive, de phonétique, de rythme et d'intonation seront proposés, de même que l'apprentissage de comptines, de poésie, de chansons, etc. La compréhension de ces supports, de même que celle de dialogues ou des divers textes proposés, est également abordable par tous.

Il en est de même au niveau de l'expression, où le même travail peut être proposé à tous les élèves.

Il paraît également important de proposer à tous un travail de repérage pour la compréhension de la société d'accueil :

- Repères spatiaux sur un environnement de plus en plus largede la classe au collège, au quartier..., au monde), qui constitueront un premier travail de géographie carte, plan), ou de géométrie.

- Repères temporels, toujours à partir de leur vécu,datations, leur histoire personnelle..., l'histoire.

- Repères sociaux et civilisationnels : repérer les divers rôles sociaux, les codes et les valeurs de la société d'accueil, leur convergence et leur divergence avec ceux de la société d'origine.

Ce travail de structuration passe bien entendu par la langue. La démarche tendra à passer de la simple désignation du réel à des niveaux d'abstraction de plus en plus fins, qui permettront un passage plus aisé vers l'acquisition de la langue scolaire et des langues et discours des diverses disciplines.

\subsection{Les activités différenciées}

\subsubsection{L'autonomie}

Chaque élève, en fonction de ses compétences et de son projet scolaire, aura à fournir un travail particulier, lié au programme de sa classe de rattachement, à ses difficultés personnelles, et aux exigences de la remise à niveau.

Pour ce faire, il faut impérativement encourager l'autonomie de l'élève, l'aider à organiser ses apprentissages, à combler ses lacunes.

\subsubsection{L'alphabétisation}

27 L'alphabétisation des élèves est une des difficultés que posent les rejoignants en collège. Les méthodes existantes ne sont pas adaptées à ces publics. L'apprentissage de la lectureécriture devra se faire en liaison étroite avec les activités de la classe et le vécu de l'élève. Ceci implique une phase de discrimination visuelle globale, relayée bien entendu par une approche syllabique systématique. Cette systématisation est importante pour des élèves 
n'ayant pas toujours à leur disposition le lexique de base du français, et ne pouvant donc émettre, dans tous les cas, des hypothèses sur les mots. est illusoire de leur demander de faire des exposés tant oraux qu'écrits, à partir de supports exclusivement écrits. Il est donc important de trouver des activités faisant le lien entre restitution orale et production écrite. Un bon exemple en est le compte rendu quotidien, par un petit groupe d'élèves, du journal télévisé ou d'une actualité quelconque. D'oral pendant quelque temps, ce compte rendu peut devenir écrit. Ce travail permet un effort de compréhension autonome d'un message difficile, l'effort de mémorisation d'une série d'informations, d'un lexique, de structures textuelles et phrastiques, la mise à distance d'un contenu informatif, l'analyse et le découpage, d'abord collectifs, du texte, sont un ensemble d'activités complexes dont la maîtrise facilite le passage à l'écrit, pour un compte rendu ou un autre exercice.

\subsubsection{Enseignement-apprentissage du métalangage des disciplines}

32 Il ne saurait être question de surseoir à l'introduction du métalangage des disciplines, sous prétexte que les performances linguistiques et communicatives sont trop faibles. En effet, de la même façon qu'il faut exposer les élèves à un lexique et à des structures riches et variées, il est important d'introduire rapidement le métalangage correct en usage dans les classes de collège.

En français par exemple, on s'efforcera de discriminer les divers types d'activités et d'introduire le métalangage grammatical :

- la grammaire concerne la phrase, tous les mots de la phrase, leur nature ou catégorie grammaticale, ou espèce de mot), leur fonction, leur genre et nombre...

- laconjugaison concerne le verbe. On nommera les formes du verbe, les temps simples ou composés, en explicitant le sens de ces termes), les modes, les personnes.

Chaque activité doit être restituée dans le champ qui l'intéresse. Cela permet une meilleure organisation des connaissances, une meilleure compréhension du système linguistique et de son fonctionnement, et une mobilisation des connaissances plus efficace. 

base de recherche à exploiter et à approfondir. Malgré les réserves exprimées par J.-P. Cuq, à nos yeux il est clair que pour les élèves francophones en difficulté, l'apprentissage $\mathrm{du}$ français scolaire relève de la même problématique que l'apprentissage d'une langue seconde pour les rejoignants.

\section{BIBLIOGRAPHIE}

ABDALLAH-PRETCEILLE M., Des enfants non-francophones à l'école ; quel apprentissage ? quel français? Paris : A. Colin, 1982.

ANDRE A., « Enfants de travailleurs immigrés : attitudes et représentations dans le corps enseignant : points de repère pour la formation ", in Migrants-formation, n 58, sept. 1984.

CUQ J.-P., Le français langue seconde. Paris : Hachette 1991.

GUMPERZ J., Sociolinguistique interactionnelle, Université de la Réunion, URA 1041 du CNRS., L'Harmattan.

LAACHER S., « 'L'école et ses miracles', Note sur les déterminants sociaux des trajectoires des enfants de familles immigrées », in Politix, n 12, 1990.

LAMRANI A., «La scolarisation des rejoignants en collège : quelles compétences développer? Quelles langues enseigner ? Comment l'enseigner? ", in Travaux de didactique, $\mathrm{n}^{\circ} 33$, Université $\mathrm{P}$. Valéry, Montpellier III, nov. 1994.

LAPARRA M., « Problèmes rencontrés lors de l'écriture de textes explicatifs en collège », in Pratiques, $\mathrm{n}^{\circ}$ 77, sept. 1993.

LAPARRA M., « Problèmes de lecture posés par l'écriture des textes historiques à visée didactique », in Pratiques, $n^{\circ}$ 71, sept. 1991.

NONNON E., « Les difficultés de langage des enfants de l'immigration in Difficultés du langage oral et écrit chez les enfants de l'immigration en échec scolaire : quelques éléments d'analyse. », in Revue Enfance, n 4, 1991.

NONNON E., « Postulat de cohérence et exigence didactique. Travailler autour des mouvements discursifs ", in Le français aujourd'hui, n 101, mars 1993. 
NONNON E., " Mettre au tableau, mettre en tableaux, ou comment structurer les discussions d'enfants. Logique naturelle et formalisations écrites », in ELA,n 81, 1991.

VIGNER G., « Le français langue de scolarisation », in ELA, numéro spécial, oct.-déc. 1992.

\section{NOTES}

1. Cf. E. NONNON : Les difficultés de langage des enfants de l'immigration in Difficultés du langage oral et écrit chez les enfants de l'immigration en échec scolaire : quelques éléments d'analyse in Enfance ${ }^{\circ}$ 4, 1991.

2. Conformément à la circulaire ministérielle de 1986 stipulant que chaque élève-rejoignant doit être inscrit dans une classe correspondant à sa classe d'âge.

3. L'animateur étant rétribué par le FAS (Fonds d'Action Sociale)

4. L'arrivée de l'élève-rejoignant en France est souvent motivée par la limite d'âge imposée par la loi en matière de regroupement familial: au-delà de seize ans, l'enfant n'a plus le droit d'émigrer ou du moins, il ne peut plus bénéficier des avantages attachés à la politique du regroupement familial.

5. Concernant le concept de langue seconde (L2), voir J.-P. CUQ: Le français langue seconde: Hachette, 1991.

6. J. GUMPERZ : « ... l'apprentissage, pour être efficace dans la communication quotidienne des immigrants culturellement et linguistiquement distincts, est fonction à la fois de l'exposition effective à la nouvelle langue que forment les locuteurs dans leur nouveau cadre. Si les immigrants s'installent en groupes fermés, si leurs relations à la maison et au travail excluent ceux qui ont un contexte de communication différent, alors l'apprentissage d'une langue nouvelle n'éliminera pas nécessairement les caractères linguistiques qui distinguent ce groupe. De nouvelles conventions pragmatiques spécifiques au groupe apparaîtront, qui continueront à affecter le système de signification des immigrants, et par conséquent leur relation avec autrui. » in Sociolinguistique interactionnelle. Université de la Réunion, URA 1041 du CNRS. - L'Harmattan.

7. G. VIGNER : Le français langue de scolarisation in ELA 10/12/1992. - p. 49.

\section{RÉSUMÉS}

La scolarisation des enfants de migrants pose le problème de l'acquisition de la langue scolaire et du métalangage des disciplines. Les difficultés d'enseignement - apprentissage que rencontrent élèves - rejoignants et enseignants sont à rapprocher de celles, plus générales, qui touchent les enfants francophones en situation d'échec scolaire. Des solutions pédagogiques doivent être mise en place : l'article propose quelques pistes de réflexions.

The instruction of immigrant/migrant children poses the problem of classroom language and of the metalanguage of the various disciplines taught at school. The learning difficulties students joining their parents in a foreign land face and the instructional difficulties their teachers face are to be likened to the broader ones which affect french speaking students in the situation of failing in school. Pedagogical solutions must be implemented. The article proposes a few reflections along various lines of thought. 
INDEX

Mots-clés : élève rejoignant, langue scolaire, langue seconde, métalangue

Keywords : classroom language, metalanguage, second language, student joining parents in a foreign land

\section{AUTEUR}

\section{ANNIE BEAUCOURT}

Maître de conférences, université Toulouse - Le Mirail, collaboratrice du C.E.I.D.R.E., IUFM de Montpellier 